\title{
Joint Generative Model for fMRI/DWI and Its Application to Population Studies
}

\author{
Archana Venkataraman ${ }^{1}$, Yogesh Rathi ${ }^{2}$, Marek Kubicki ${ }^{2}$, \\ Carl-Fredrik Westin ${ }^{3,1}$, and Polina Golland ${ }^{1}$ \\ 1 MIT Computer Science and Artificial Intelligence Laboratory, Cambridge, MA \\ 2 Psychiatry Neuroimaging Laboratory, Harvard Medical School, Boston, MA \\ 3 Laboratory for Mathematics Imaging, Harvard Medical School, Boston, MA
}

\begin{abstract}
We propose a novel probabilistic framework to merge information from DWI tractography and resting-state fMRI correlations. In particular, we model the interaction of latent anatomical and functional connectivity templates between brain regions and present an intuitive extension to population studies. We employ a mean-field approximation to fit the new model to the data. The resulting algorithm identifies differences in latent connectivity between the groups. We demonstrate our method on a study of normal controls and schizophrenia patients.
\end{abstract}

\section{Introduction}

The interaction between functional and anatomical connectivity provides a rich framework for understanding the brain. Functional connectivity is commonly measured via temporal correlations in resting-state fMRI data. These correlations are believed to reflect the intrinsic functional organization of the brain [1]. Anatomical connectivity is often measured using DWI tractography, which estimates the configuration of underlying white matter fibers [2. In this work we propose and demonstrate a novel probabilistic framework to infer the relationship between these modalities. The model is based on latent connectivities between brain regions and makes intuitive assumptions about the data generation process. We present a natural extension of the model to population studies, which we use to identify widespread connectivity changes in schizophrenia.

To date, relatively little progress has been made in fusing information between the aforementioned anatomical and functional modalities. It has been shown that while a high degree of structural connectivity predicts higher functional correlations, the converse does not always hold 34. For example, strong functional correlations can be found between spatially distributed locations in the brain. However, one is more likely to identify white matter tracts connecting nearby regions. Graph-theoretic models have previously been used to examine the correspondence between independently estimated structural hubs and functional networks [56]. In contrast, we infer a population template of connectivity using both resting-state fMRI correlations and DWI tractography.

We demonstrate the capability of our model to learn stable patterns on a population study of schizophrenia. Schizophrenia is a poorly-understood disorder

T. Jiang et al. (Eds.): MICCAI 2010, Part I, LNCS 6361, pp. 191-199, 2010

(C) Springer-Verlag Berlin Heidelberg 2010 


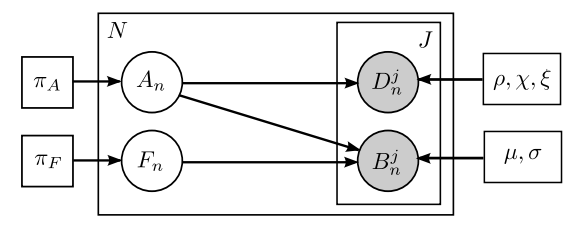

(a) Joint fMRI/DWI model

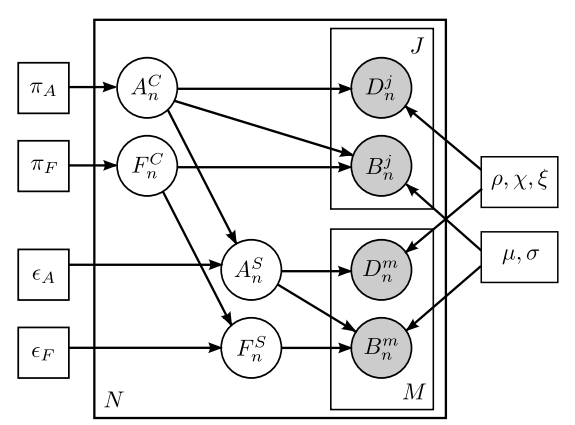

(b) Model of population differences

Fig. 1. (a) Joint connectivity model for a single population. (b) Joint model for the effects of schizophrenia. The pairwise connections are indexed by $n=1, \ldots, N$, the control subjects are indexed by $j=1, \ldots, J$, and the schizophrenia patients are indexed by $m=1, \ldots, M$. Squares indicate non-random parameters; circles indicate hidden random variables; all shaded variables are observed.

marked by widespread cognitive difficulties affecting intelligence, memory, and executive attention. These impairments are not localized to a particular cortical region; rather, they reflect abnormalities in widely-distributed functional and anatomical networks [7/8. In accordance with these findings, our model identifies connectivity differences in spatially extensive networks.

Only a few studies to date have combined resting-state fMRI and DWI tractography to analyze schizophrenia 910. Univariate statistical tests are commonly used to identify significant population differences in temporal correlations and in mean Fractional Anisotrophy (FA) values. The relevant connections are then compared across modalities to draw conclusions. This approach treats functional and structural connections as a priori independent and ignores distributed patterns of connectivity. In contrast, our model jointly infers the entire pattern of functional and anatomical connectivity, as well as the group differences.

\section{Generative Model and Inference}

Unlike voxel- and ROI-based analysis, we model the behavior of pairwise connections between regions of the brain. Our observed variables are correlations in resting-state fMRI and average FA values along the white matter tracts.

Latent Connectivity. Fig. 1(a) shows our model for a single population. Let $N$ be the total number of relevant connections in the brain. $A_{n}$ and $F_{n}$ are the latent anatomical and functional connectivity measures between the two regions associated with the $n^{\text {th }}$ connection. $A_{n}$ is a binary random variable with parameter $\pi_{A}: P\left(A_{n} ; \pi_{A}\right)=\pi_{A}^{A_{n}}\left(1-\pi_{A}\right)^{1-A_{n}}$. It indicates the presence or absence of a direct anatomical pathway between the regions. In contrast, $F_{n}$ 
is a tri-state random variable drawn from a multinomial distribution $\pi_{F}$. These states represent little or no functional co-activation $\left(F_{n}=0\right)$, positive functional synchrony $\left(F_{n}=1\right)$, and negative functional synchrony $\left(F_{n}=-1\right)$ between the regions. For notational convenience, we represent $F_{n}$ as a length-three indicator vector with exactly one of its elements $\left\{F_{n k}: k=-1,0,1\right\}$ equal to one:

$$
P\left(F_{n} ; \pi_{F}\right)=\prod_{k=-1}^{1}\left(\pi_{F k}\right)^{F_{n k}}
$$

Data Likelihood. Let $J$ be the number of subjects. The DWI measurement $D_{n}^{j}$ for the $j^{t h}$ subject is a noisy observation of the anatomical connectivity $A_{n}$ :

$$
\begin{aligned}
P\left(D_{n}^{j} \mid A_{n} ;\{\rho, \chi, \xi\}\right)= & {\left[\rho_{0} \delta\left(D_{n}^{j}\right)+\left(1-\rho_{0}\right) \mathcal{N}\left(D_{n}^{j} ; \chi_{0}, \xi_{0}^{2}\right)\right]^{\left(1-A_{n}\right)} } \\
& \cdot\left[\rho_{1} \delta\left(D_{n}^{j}\right)+\left(1-\rho_{1}\right) \mathcal{N}\left(D_{n}^{j} ; \chi_{1}, \xi_{1}^{2}\right)\right]^{A_{n}}
\end{aligned}
$$

where $\delta(\cdot)$ is the Dirac delta function, $N\left(\cdot ; \chi, \xi^{2}\right)$ is a Gaussian distribution with mean $\chi$ and variance $\xi^{2}$, and $\rho$ is the probability of failing to find a tract between two regions. The value zero of $D_{n}^{j}$ is arbitrarily chosen to represent "No Connection". $D_{n}^{j}$ is strictly positive when a connection is present.

The BOLD fMRI correlation $B_{n}^{j}$ for the $j^{t h}$ subject depends on both $F_{n}$ and $A_{n}$ since direct anatomical connections predict higher functional correlations:

$$
P\left(B_{n}^{j} \mid A_{n}, F_{n} ;\{\mu, \sigma\}\right)=\prod_{k=-1}^{1}\left[\mathcal{N}\left(B_{n}^{j} ; \mu_{0 k}, \sigma_{0 k}^{2}\right)^{\left(1-A_{n}\right)} \cdot \mathcal{N}\left(B_{n}^{j} ; \mu_{1 k}, \sigma_{1 k}^{2}\right)^{A_{n}}\right]^{F_{n k}} .
$$

Using histograms of the data, we verified that the Gaussian distributions in Eqs. (22) provide reasonable approximations for the DWI and fMRI data. Pragmatically, they greatly simplify the learning/inference steps.

Population Differences. Fig. 1(b) depicts an extension of the model to a population study involving controls and schizophrenia patients. We model differences between the groups within the latent connectivities alone and share the data likelihood distributions between the two populations.

We treat the latent connectivity templates $\left\{A_{n}^{S}, F_{n}^{S}\right\}$ of the schizophrenia population as "corrupted" versions of the healthy template. In particular, with (small) probability $\epsilon$, each connection can switch its state:

$$
\begin{aligned}
& P\left(A_{n}^{S} \mid A_{n}^{C} ; \epsilon_{A}\right)=\epsilon_{A}^{A_{n}^{C}\left(1-A_{n}^{S}\right)+\left(1-A_{n}^{C}\right) A_{n}^{S}} \cdot\left(1-\epsilon_{A}\right)^{A_{n}^{C} A_{n}^{S}+\left(1-A_{n}^{C}\right)\left(1-A_{n}^{S}\right)}, \\
& P\left(F_{n}^{S} \mid F_{n}^{C} ; \epsilon_{F}\right)=\prod_{k=-1}^{1} \frac{\epsilon}{F}^{\left(1-F_{n k}^{C} F_{n k}^{S}\right)} \cdot\left(1-\epsilon_{F}\right)^{F_{n k}^{C} F_{n k}^{S}} .
\end{aligned}
$$

For robustness, we rely on a single scalar to govern the probability of change within each modality. Additionally, in Eq. (5) we assume that functional connectivity switches to its other two states with equal probability. 
Variational EM Solution. It is not difficult to show that the complete loglikelihood of all the random variables has multiplicative interactions among the hidden variables. For this reason, we employ the mean-field algorithm 11 to approximate the posterior probability distribution of the latent variables using a fully factorized distribution.

We let $\left\{\hat{p}_{n}^{C}, \hat{p}_{n}^{S}, \hat{q}_{n k}^{C}, \hat{q}_{n k}^{S}\right\}$ represent the posterior probability estimates for $\left\{A_{n}^{C}, A_{n}^{S}, F_{n}^{C}, F_{n}^{S}\right\}$. The variational EM algorithm alternates between updating the posterior estimates and the model parameters to minimize the variational free energy. Due to space constraints, we directly present the update rules.

Learning. We fix the posterior distributions and learn the model parameters. Let

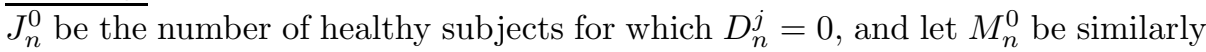
defined for schizophrenia patients. The update rules are identical to those of the standard EM. The probability estimates are sums of the latent posteriors:

$$
\begin{array}{ll}
\pi_{A}=\frac{1}{N} \sum_{n=1}^{N} \hat{p}_{n}^{C} & \pi_{F k}=\frac{1}{N} \sum_{n=1}^{N} \hat{q}_{n k}^{C} \\
\epsilon_{A}=\frac{1}{N} \sum_{n=1}^{N} \hat{p}_{n}^{C}\left(1-\hat{p}_{n}^{S}\right)+\left(1-\hat{p}_{n}^{C}\right) \hat{p}_{n}^{S} & \epsilon_{F}=1-\frac{1}{N} \sum_{n=1}^{N} \sum_{k=-1}^{1} \hat{q}_{n k}^{C} \hat{q}_{n k}^{S}
\end{array}
$$

and the density parameters are equal to weighted statistics of the data:

$$
\begin{aligned}
\mu_{1 k} & =\frac{\sum_{n=1}^{N}\left[\hat{p}_{n}^{C} \hat{q}_{n k}^{C} \sum_{j=1}^{J} B_{n k}^{j}+\hat{p}_{n}^{S} \hat{q}_{n k}^{S} \sum_{m=1}^{M} B_{n k}^{m}\right]}{\sum_{n=1}^{N}\left[\hat{p}_{n}^{C} \hat{q}_{n k}^{C} J+\hat{p}_{n}^{S} \hat{q}_{n k}^{S} M\right]} \\
\sigma_{1 k}^{2} & =\frac{\sum_{n=1}^{N}\left[\hat{p}_{n}^{C} \hat{q}_{n k}^{C} \sum_{j=1}^{J}\left(B_{n k}^{j}-\mu_{1 k}\right)^{2}+\hat{p}_{n}^{S} \hat{q}_{n k}^{S} \sum_{m=1}^{M}\left(B_{n k}^{m}-\mu_{1 k}\right)^{2}\right]}{\sum_{n=1}^{N}\left[\hat{p}_{n}^{C} \hat{q}_{n k}^{C} J+\hat{p}_{n}^{S} \hat{q}_{n k}^{S} M\right]} \\
\rho_{1} & =\frac{\sum_{n=1}^{N}\left[\hat{p}_{n}^{C} J_{n}^{0}+\hat{p}_{n}^{S} M_{n}^{0}\right]}{\sum_{n=1}^{N}\left[\hat{p}_{n}^{C} J+\hat{p}_{n}^{S} M\right]} \quad \chi_{1}=\frac{\sum_{n=1}^{N}\left[\hat{p}_{n}^{C} \sum_{j: D_{n}^{j}>0} D_{n}^{j}+\hat{p}_{n}^{S} \sum_{m: D_{n}^{m}>0} D_{n}^{m}\right]}{\sum_{n=1}^{N}\left[\hat{p}_{n}^{C}\left(J-J_{n}^{0}\right)+\hat{p}_{n}^{S}\left(M-M_{n}^{0}\right)\right]} \\
\xi_{1}^{2} & =\frac{\sum_{n=1}^{N}\left[\hat{p}_{n}^{C} \sum_{j: D_{n}^{j}>0}\left(D_{n}^{j}-\chi_{1}\right)^{2}+\hat{p}_{n}^{S} \sum_{m: D_{n}^{m}>0}\left(D_{n}^{m}-\chi_{1}\right)^{2}\right]}{\sum_{n=1}^{N}\left[\hat{p}_{n}^{C}\left(J-J_{n}^{0}\right)+\hat{p}_{n}^{S}\left(M-M_{n}^{0}\right)\right]}
\end{aligned}
$$

The parameter updates for $\left\{\mu_{0 k}, \sigma_{0 k}^{2}, \rho_{0}, \chi_{0}, \xi_{0}^{2}\right\}$ are trivially obtained from these expressions by replacing $\hat{p}_{n}^{C}$ with $\left(1-\hat{p}_{n}^{C}\right)$ and $\hat{p}_{n}^{S}$ with $\left(1-\hat{p}_{n}^{S}\right)$.

Inference. We fix the model parameters and update the variational posteriors. $\overline{\text { We use } \mathcal{P}_{l}}(\cdot)$ to denote the mixture distribution in Eq. (2) and $\mathcal{G}_{l k}(\cdot)$ to denote a normal distribution with parameters $\left\{\mu_{l k}, \sigma_{l k}\right\}$ in order to obtain:

$$
\begin{aligned}
& \frac{\hat{p}_{n}^{C}}{1-\hat{p}_{n}^{C}}=\left(\frac{\pi_{A}}{1-\pi_{A}}\right)\left(\frac{1-\epsilon_{A}}{\epsilon_{A}}\right)^{\left(2 \hat{p}_{n}^{S}-1\right)} \prod_{j=1}^{J}\left(\frac{\mathcal{P}_{1}\left(D_{n}^{j}\right)}{\mathcal{P}_{0}\left(D_{n}^{j}\right)}\right) \prod_{k=-1}^{1}\left(\frac{\mathcal{G}_{1 k}\left(B_{n}^{j}\right)}{\mathcal{G}_{0 k}\left(B_{n}^{j}\right)}\right)^{\hat{q}_{n k}^{C}} \\
& \frac{\hat{p}_{n}^{S}}{1-\hat{p}_{n}^{S}}=\left(\frac{1-\epsilon_{A}}{\epsilon_{A}}\right)^{\left(2 \hat{p}_{n}^{C}-1\right)} \prod_{m=1}^{M}\left(\frac{\mathcal{P}_{1}\left(D_{n}^{m}\right)}{\mathcal{P}_{0}\left(D_{n}^{m}\right)}\right) \prod_{k=-1}^{1}\left(\frac{\mathcal{G}_{1 k}\left(B_{n}^{m}\right)}{\mathcal{G}_{0 k}\left(B_{n}^{m}\right)}\right)^{\hat{q}_{n k}^{S}}
\end{aligned}
$$




$$
\begin{array}{ll}
\hat{q}_{n k}^{C} \propto \pi_{F k} \cdot\left(\frac{1-\epsilon_{F}}{\left(\epsilon_{F} / 2\right)}\right)^{\hat{q}_{n k}^{S}} \prod_{j=1}^{J}\left(\mathcal{G}_{1 k}\left(B_{n}^{j}\right)\right)^{\hat{p}_{n}^{C}}\left(\mathcal{G}_{0 k}\left(B_{n}^{j}\right)\right)^{\left(1-\hat{p}_{n}^{C}\right)} & \text { s.t. } \sum_{k=-1}^{1} \hat{q}_{n k}^{C}=1 \\
\hat{q}_{n k}^{S} \propto\left(\frac{1-\epsilon_{F}}{\left(\epsilon_{F} / 2\right)}\right)^{\hat{q}_{n k}^{C}} \prod_{m=1}^{M}\left(\mathcal{G}_{1 k}\left(B_{n}^{m}\right)\right)^{\hat{p}_{n}^{S}}\left(\mathcal{G}_{0 k}\left(B_{n}^{m}\right)\right)^{\left(1-\hat{p}_{n}^{S}\right)} & \text { s.t. } \sum_{k=-1}^{1} \hat{q}_{n k}^{S}=1
\end{array}
$$

As seen, the updates can be decomposed into a prior term (for normal subjects only), a term arising from the connectivity changes between the populations, and a data likelihood term involving the other modality.

Model Evaluation. Based on the latent posterior probabilities $\left\{\hat{p}_{n}^{C}, \hat{p}_{n}^{S}, \hat{q}_{n k}^{C}, \hat{q}_{n k}^{C}\right\}$, the empirical probability of change in the anatomical or functional connectivity of the $n^{\text {th }}$ connection is

$$
\hat{\epsilon}_{A}^{n}=\hat{p}_{n}^{C}\left(1-\hat{p}_{n}^{S}\right)+\left(1-\hat{p}_{n}^{S}\right) \hat{p}_{n}^{S} \quad \text { and } \quad \hat{\epsilon}_{F}^{n}=1-\sum_{k=-1}^{1} \hat{q}_{n k}^{C} \hat{q}_{n k}^{S}
$$

respectively. We evaluate the significance and robustness of our model through non-parametric permutation tests and cross-validataion. To construct the distributions for $\hat{\epsilon}_{A}^{n}$ and $\hat{\epsilon}_{F}^{n}$ under the null hypothesis, we randomly permute the subject labels (NC vs. SZ) 10,000 times. For each permutation, we fit the model and compute the relevant statistics in Eq. (6). The significance (p-value) of each connection is equal to the proportion of permutations for which the computed statistic is greater than or equal to the value obtained under the true labeling.

We also quantify the model's predictive power via ten-fold cross validation. The model is fit using the training subjects, and a likelihood ratio test is used to predict the diagnosis for the held-out group. The data is resampled 20 times to ensure stability of the results. For comparison, we perform the same ten-fold cross validation using support vector machine (SVM) classifiers trained on the fMRI correlations, the DWI FA values, and the combined fMRI and DWI data.

\section{Results}

Data. We demonstrate our model on a study of 18 male patients with chronic schizophrenia and 18 male healthy controls. The control participants were group matched to the patients on age, handedness, parental socioeconomic status, and an estimated premorbid IQ. For each subject, an anatomical scan (SPGR, $T R=$ $7.4 s, T E=3 \mathrm{~ms}, F O V=26 \mathrm{~cm}^{2}$, res $=1 \mathrm{~mm}^{3}$ ), a diffusion-weighted scan (EPI, $T R=17 \mathrm{~s}, \mathrm{TE}=78 \mathrm{~ms}, \mathrm{FOV}=24 \mathrm{~cm}^{2}$, res $=1.66 \times 1.66 \times 1.7 \mathrm{~mm}, 51$ gradient directions with $b=900 \mathrm{~s} / \mathrm{mm}^{2}, 8$ baseline scans with $b=0 \mathrm{~s} / \mathrm{mm}^{2}$ ) and a restingstate functional scan (EPI-BOLD, $T R=3 \mathrm{~s}, T E=30 \mathrm{~ms}, F O V=24 \mathrm{~cm}^{2}$, $r e s=1.875 \times 1.875 \times 3 \mathrm{~mm}$ ) were acquired using a $3 \mathrm{~T}$ GE Echospeed system.

Pre-Processing. We segmented the structural images into 77 anatomical regions with Freesurfer [12]. The DWI data was corrected for eddy-current distortions. Two-tensor tractography was used to estimate the white matter fibers [13]. We compute the DWI connectivity $D_{n}^{j}$ by averaging FA along all fibers connecting the corresponding regions. If no tracts are found, $D_{n}^{j}$ is set to zero. 
Table 1. Parameters of the joint model in Fig. 11(a), estimated separately for control $(\mathrm{NC})$ and schizophrenic (SZ) populations, and for the entire dataset $\left.(\mathrm{NC}+\mathrm{SZ})^{1}\right]$

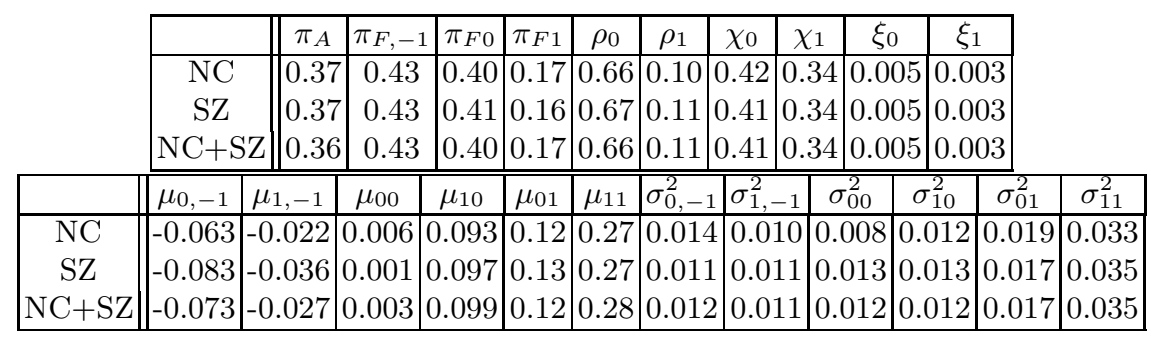

We discarded the first five fMRI time points and performed motion correction by rigid body alignment and slice timing correction using FSL [14]. The data was spatially smoothed using a Gaussian filter, temporally low-pass filtered with $0.08 \mathrm{~Hz}$ cutoff, and motion corrected via linear regression. Finally, we regressed out global contributions to the timecourses from the white matter, ventricles and the whole brain. We extract the fMRI connectivity $B_{n}^{j}$ by computing Pearson correlation coefficients between every pair of voxels in the two regions of the $n^{\text {th }}$ connection, applying the Fisher-r-to-z transform to each correlation (to enforce normality), and averaging these values. Since our anatomical regions are large, the correlation between the mean timecourses of two regions shows poor correspondence with the distribution of voxel-wise correlations between them. We believe our measure is more appropriate for assessing fMRI connectivity.

To inject prior clinical knowledge, we pre-selected 8 brain structures (corresponding to 16 regions) that are believed to play a role in schizophrenia: the superior temporal gyrus, rostral middle frontal gyrus, hippocampus, amygdala, posterior cingulate, rostral anterior cingulate, parahippocampal gyrus, and transverse temporal gyrus. We model only the $1096\left(16 \times 76-\left(\begin{array}{c}16 \\ 2\end{array}\right)\right)$ unique pairwise connections between these ROIs and all other regions in the brain.

Joint Connectivity Model. We first fit the joint model in Fig. 1(a) to each population separately as well as to the entire dataset. Table 1 reports the parameters of the three models. We observe that $\{\mu, \sigma, \rho, \chi, \xi\}$ are largely consistent across the three models. This supports our hypothesis that group differences appear in the latent connectivities rather than in the data likelihood parameters.

Population Study. Fig. 2 depicts the significantly different $(p<0.05, \hat{\epsilon}>0.5)$ anatomical and functional connections identified by the algorithm. Table 2 lists the corresponding regions and p-values. Due to space limitations, we report just the 3 connections with the smallest p-values in each modality.

As seen from Fig. 2(b), schizophrenia patients exhibit increased functional connectivity between the parietal/posterior cingulate region and the frontal lobe and reduced functional connectivity between the parietal/posterior cingulate region and the temporal lobe. These results confirm the hypotheses of widespread

${ }^{1} \chi_{0} \chi_{1}$ implies that spurious DWI fibers arise due to artificially high anisotropy. 


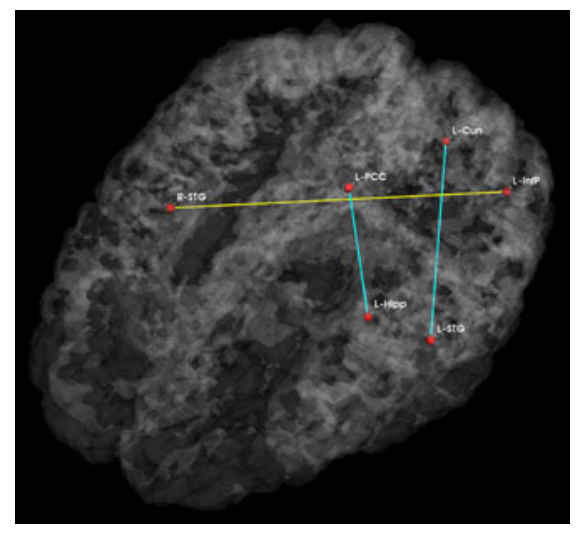

(a) Anatomical Differences

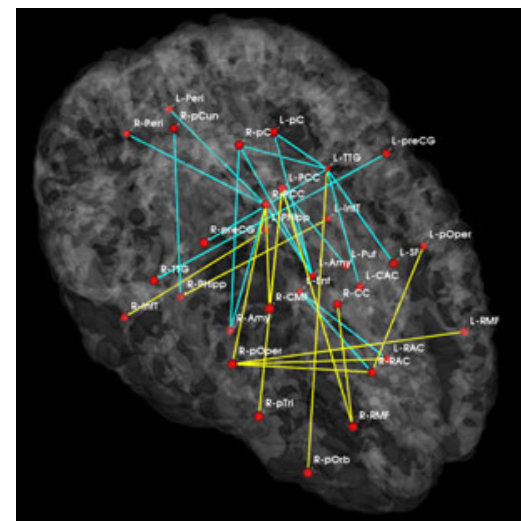

(b) Functional Differences

Fig. 2. Significant anatomical and functional connectivity differences $(p<0.05$ and $\left.\hat{\epsilon}_{A}^{n}, \hat{\epsilon}_{F}^{n}>0.5\right)$. Blue lines indicate higher connectivity in the control group; yellow lines indicate higher connectivity in the schizophrenia population.

Table 2. Top 5 significant anatomical (top) and functional (bottom) connections

\begin{tabular}{|l|l|c|c|}
\hline Region 1 & Region 2 & $\mathrm{p}$-value & $\hat{\epsilon}_{A}^{n} / \hat{\epsilon}_{F}^{n}$ \\
\hline R Superior Temporal Gyrus (R-STG) & L Inferior Parietal (L-InfP) & 0.0005 & 0.85 \\
L Posterior Cingulate (L-PCC) & L Hippocampus (L-Hipp) & 0.0045 & 0.79 \\
L Superior Temporal Gyrus (L-STG) & L Cuneus (L-Cun) & 0.011 & 0.93 \\
\hline R Pars Triangularis (R-pTri) & L Posterior Cingulate (L-PCC) & 0.0001 & 0.92 \\
R Paracentral Gyrus (R-pC) & L Transverse Temporal (L-TTG) & 0.0001 & 0.89 \\
L Transverse Temporal (L-TTG) & L Paracentral Gyrus (L-pC) & 0.0001 & 0.55 \\
\hline
\end{tabular}

functional connectivity changes in schizophrenia and of functional abnormalities involving the default network.

The differences in anatomical connectivity implicate the superior temporal gyrus and hippocampus. These regions have been cited in prior DTI studies of schizophrenia [15]. We note that relatively few anatomical connections exhibit significant differences between the two populations. This may stem from our choice of ROIs. In particular, we rely on Freesurfer parcellations, which provide anatomically meaningful correspondences across subjects. These larger regions also mitigate the effects of minor registration errors. However, they may be too big to capture structural differences between the groups. We emphasize that our model can be easily applied to finer scale parcelations in future studies.

Table 3 reports classification accuracies for the generative model and SVM classifiers. Despite not being optimized for classification, our model exhibits above-chance generalization accuracy. We note that even the SVM does not achieve high discrimination accuracy. This underscores the well-documented challenge of finding robust functional and anatomical changes induced by schizophrenia [15]. We stress that our main goal is to explain differences in connectivity. Classification is only presented for validation. 
Table 3. Training and testing accuracy of ten-fold cross validation for the control (NC) and Schizophrenic (SZ) populations

\begin{tabular}{|c|c|c|c|c|}
\hline & Training NC & Training SZ & Testing NC & Testing SZ \\
\hline Joint fMRI/DWI Model & $0.99 \pm 0.005$ & $0.88 \pm 0.01$ & $0.61 \pm 0.06$ & $0.55 \pm 0.05$ \\
linear SVM fMRI & $1.00 \pm 0.00$ & $1.00 \pm 0.00$ & $0.54 \pm 0.05$ & $0.61 \pm 0.05$ \\
linear SVM DWI & $1.00 \pm 0.00$ & $1.00 \pm 0.00$ & $0.59 \pm 0.08$ & $0.58 \pm 0.06$ \\
linear SVM fMRI/DWI & $1.00 \pm 0.00$ & $1.00 \pm 0.00$ & $0.67 \pm 0.04$ & $0.60 \pm 0.05$ \\
\hline
\end{tabular}

\section{Conclusion}

We proposed a novel probabilistic framework that fuses information from restingstate fMRI data and DWI tractography. We further extended the basic approach to model connectivity differences between two populations. We show that our method captures changes in functional and anatomical connectivity induced by schizophrenia. In particular, we detect increased functional connectivity from the parietal lobe to the frontal lobe and decreased functional connectivity from the parietal lobe to the temporal lobe. We also find significant anatomical connectivity differences involving the superior temporal gyrus, the posterior cingulate and the hippocampus. Finally, we demonstrate the predictive power of our model through cross validation. These results establish the promise of our approach for combining multiple imaging modalities to better understand the brain.

Acknowledgments. This work was supported in part by the National Alliance for Medical Image Analysis (NIH NIBIB NAMIC U54-EB005149), the Neuroimaging Analysis Center (NIH NCRR NAC P41-RR13218), the NSF CAREER Grant 0642971 and NIH R01MH074794. A. Venkataraman is supported by the National Defense Science and Engineering Graduate Fellowship (NDSEG).

\section{References}

1. Buckner, R.L., Vincent, J.L.: Unrest at rest: Default activity and spontaneous network correlations. NeuroImage 37(4), 1091-1096 (2007)

2. Basser, P., Pierpaoli, C.: Microstructural and physiological features of tissues elucidated by quantitative-diffusion-tensor mri. Journal of Magnetic Resonance, Series B 111, 209-219 (1996)

3. Honey, C., et al.: Predicting human resting-state functional connectivity from structural connectivity. PNAS 106(6), 2035-2040 (2009)

4. Koch, M.A., et al.: An investigation of functional and anatomical connectivity using magnetic resonance imaging. NeuroImage 16(1), 241-250 (2002)

5. Sporns, O., et al.: Theoretical neuroanatomy: Relating anatomical and functional connectivity in graphs and cortical connection matrices. Cerebral Cortex 10(2), 127-141 (2000)

6. Honey, C., et al.: Network structure of cerebral cortex shapes functional connectivity on multiple time scales. PNAS 104(24), 10240-10245 (2007) 
7. Gabrieli-Whitfield, S., et al.: Hyperactivity and hyperconnectivity of the default network in schizophrenia and in first-degree relatives of persons with schizophrenia. PNAS 106(4), 1279-1284 (2009)

8. Burns, J., et al.: Structural disconnectivity in schizophrenia: A diffusion tensor magnetic resonance imaging study. Br. J. Psychiatry 182, 439-443 (2003)

9. Ke, M., et al.: Combined analysis for resting state fmri and dti data reveals abnormal development of function-structure in early-onset schizophrenia. In: Wang, G., Li, T., Grzymala-Busse, J.W., Miao, D., Skowron, A., Yao, Y. (eds.) RSKT 2008. LNCS (LNAI), vol. 5009, pp. 628-635. Springer, Heidelberg (2008)

10. Zhou, Y., et al.: Altered resting-state functional connectivity and anatomical connectivity of hippocampus in schizophrenia. Schiz. Res. 100(1-3), 120-132 (2008)

11. Jordan, M., et al.: An introduction to variational methods for graphical models. Machine Learning 37(2), 183-233 (1999)

12. Fischl, B., et al.: Sequence-independent segmentation of magnetic resonance images. NeuroImage 23, 69-84 (2004)

13. Malcolm, J., Shenton, M., Rathi, Y.: Neural tractography using an unscented kalman filter. In: IPMI, pp. 126-138 (2009)

14. Smith, S.M., et al.: Advances in functional and structural $\mathrm{mr}$ image analysis and implementation as fsl. NeuroImage 23(51), 208-219 (2004)

15. Kubicki, M., et al.: A review of diffusion tensor imaging studies in schizophrenia. Journal of Psychiatric Research 41(1-2), 15-30 (2007) 